\title{
The California Planet Survey. II. A Saturn-Mass Planet Orbiting the M Dwarf GI 6491
}

\author{
John Asher Johnson, ${ }^{2}$ Andrew W. Howard, ${ }^{3,4}$ Geoffrey W. Marcy, ${ }^{3}$ Brendan P. Bowler,${ }^{5}$ Gregory W. Henry, ${ }^{6}$ \\ Debra A. Fischer, ${ }^{7}$ Kevin Apps, ${ }^{8}$ Howard IsAacson, ${ }^{3}$ And Jason T. Wright ${ }^{9}$ \\ Received 2009 November 7; accepted 2009 December 17; published 2010 January 26
}

\begin{abstract}
We report precise Doppler measurements of the nearby $(d=10.34$ pc) M dwarf Gl 649 that reveal the presence of a planet with a minimum mass $M_{P} \sin i=0.328 M_{\text {Jup }}$ in an eccentric $(e=0.30), 598.3$ day orbit. Our photometric monitoring reveals Gl 649 to be a new variable star with brightness changes on both rotational and decadal timescales. However, neither of these timescales are consistent with the 600 day Doppler signal and so provide strong support for planetary reflex motion as the best interpretation of the observed radial velocity variations. Gl 649b is only the seventh Doppler-detected giant planet around an M dwarf. The properties of the planet and host-star therefore contribute significant information to our knowledge of planet formation around low-mass stars. We revise and refine the occurrence rate of giant planets around M dwarfs based on the California Planet Survey sample of low-mass stars $\left(M_{\star}<0.6 M_{\odot}\right)$. We find that $f=3.4_{-0.9}^{+2.2 \%}$ of stars with $M_{\star}<0.6 M_{\odot}$ harbor planets with $M_{P} \sin i>0.3 M_{\text {Jup }}$ and $a<2.5$ AU. When we restrict our analysis to metal-rich stars with $[\mathrm{Fe} / \mathrm{H}]>+0.2$, we find that the occurrence rate is $10.7_{-4.2}^{+5.9 \%}$.
\end{abstract}

\section{INTRODUCTION}

Compared to the knowledge gleaned from the large sample of giant planets around Sunlike stars, little is known about the characteristics of Jovian planets around M dwarfs. This is due primarily to the empirical finding that the occurrence rate of detectable planets scales with stellar mass (Johnson et al. 2007); low-mass stars $\left(M_{\star}<0.6 M_{\odot}\right)$ simply do not harbor giant planets very frequently (Endl et al. 2003; Butler et al. 2006). The frequency of giant planets with $M_{P} \sin i>0.3 M_{\text {Jup }}$ around Sunlike stars is $8 \%$ within $2.5 \mathrm{AU}$ (Cumming et al. 2008), and the occurrence of giant planets around $\mathrm{M}$ dwarfs is roughly a factor of 4 lower (Johnson et al. 2007).

While the lower masses of $\mathrm{M}$ dwarfs decreases the likelihood of giant planet occurrence, a handful of Jovian planets have been discovered around low-mass stars. The sample of $\mathrm{M}$ dwarfs

\footnotetext{
${ }^{1}$ Based on observations obtained at the W. M. Keck Observatory, which is operated jointly by the University of California and the California Institute of Technology. Keck time has been granted by both NASA and the University of California.

${ }^{2}$ Department of Astrophysics, California Institute of Technology, Pasadena, CA 91125; johnjohn@astro.berkeley.edu.

${ }^{3}$ Department of Astronomy, University of California, Berkeley, CA 94720.

${ }^{4}$ Townes Fellow, Space Sciences Laboratory, University of California, Berkeley, CA 94720-7450.

${ }^{5}$ Institute for Astronomy, University of Hawai'i, Honolulu, HI 96822.

${ }^{6}$ Center of Excellence in Information Systems, Tennessee State University, Nashville, TN 37209.

${ }^{7}$ Yale Astronomy Department, New Haven, CT 06520-8101.

${ }^{8}$ Cheyne Walk Observatory, Horley, Surrey, RH6 7LR, UK.

${ }^{9}$ The Pennsylvania State University, University Park, PA 16802.
}

known to harbor at least one Doppler-detected giant planet $\left(M_{P} \sin i>0.2 M_{\text {Jup }}\right)$ is listed in Table 1 and shown in the H-R diagram in Figure 1. Also given in that table are the stellar and planetary masses from the literature, and stellar metallicities from the broadband photometric calibration of Johnson \& Apps (2009).

These planets and their host stars demonstrate that stellar mass is not the only characteristic that correlates with the probability of a star harboring a planet. Stellar metallicity has been shown to be a strong predictor of planet occurrence around Sunlike stars (Fischer \& Valenti 2005a), and the correlation between planet frequency and stellar metal content appears to hold for the M dwarfs, as well. Johnson \& Apps (2009) found that M dwarfs with Jovian planets tend to be significantly metal-rich compared to a $10 \mathrm{pc}$, volume-limited sample of stars on the lower main sequence. For example, G1849 harbors a Jovian planet in a long-period orbit and is among the most metal-rich stars in the Solar neighborhood with $[\mathrm{Fe} / \mathrm{H}]>+0.45$.

If this preliminary trend proves to be real then it will provide valuable constraints for theoretical models of planet formation around a broad range of stellar characteristics. The effect of metallicity on planet occurrence will also inform the target selection of future Doppler and transit surveys targeting lowmass stars (e.g., Irwin et al. 2008), as well as the interpretation of results from direct-imaging, astrometric, and microlensing surveys (e.g., Nielsen \& Close 2009; Pravdo \& Shaklan 2009; Dong et al. 2009).

As the time baselines, sample sizes, and Doppler precision increase for the various Doppler surveys of low-mass stars, the relationships between the physical characteristics of stars and the 
TABLE 1

Properties of M DWARFS with Giant Planets

\begin{tabular}{|c|c|c|c|c|c|c|c|}
\hline Gliese Number & Hipparcos Number & Spectral Type & $\begin{array}{c}\text { Stellar Mass } \\
\left(M_{\odot}\right)\end{array}$ & {$[\mathrm{Fe} / \mathrm{H}]$} & $\begin{array}{c}M_{P} \sin i \\
\left(M_{\text {Jup }}\right) \\
\end{array}$ & $\begin{array}{c}\text { Semimajor Axis } \\
\text { (AU) }\end{array}$ & Reference \\
\hline ......... & 113020 & M4 & 0.32 & +0.37 & $0.6189,1.9275^{\mathrm{a}}$ & $0.207,0.130$ & Rivera et al. (2005) \\
\hline$\ldots \ldots \ldots$ & 109388 & M3 & 0.45 & +0.58 & $0.83^{\mathrm{b}}$ & 2.35 & Butler et al. (2006) \\
\hline ......... & $\ldots$ & M4 & 0.24 & $\ldots$ & 1.17 & 0.95 & Johnson et al. (2007) \\
\hline$\ldots \ldots \ldots$ & 106440 & M2 & 0.45 & -0.12 & 0.64 & 3.4 & Bailey et al. (2009) \\
\hline$\ldots \ldots \ldots \ldots$ & 22627 & M4 & 0.36 & +0.30 & 0.9 & 2.42 & Howard et al. (2010) \\
\hline$\ldots \quad \ldots \ldots \ldots \ldots$ & 79431 & M3.5 & 0.50 & +0.4 & 1.1 & 0.34 & Apps et al. (2010) \\
\hline $649 \quad \ldots \ldots \ldots$ & 83043 & M1.5 & 0.54 & +0.1 & 0.328 & 1.135 & This work \\
\hline
\end{tabular}

${ }^{a}$ The Gl 876 planetary system contains two resonant Jovian planets and an inner "super Earth" with $M_{P} \sin i=5.9 M_{\oplus}$.

${ }^{\mathrm{b}}$ The orbit solution for Gl.849 includes a linear velocity trend $d v / d t=-4.7 \mathrm{~m} \mathrm{~s}^{-1} \mathrm{yr}^{-1}$, which may correspond to a second planet.

${ }^{\mathrm{c}}$ The orbit solution for G1.317 includes a linear velocity trend $d v / d t=7.6 \mathrm{~m} \mathrm{~s}^{-1} \mathrm{yr}^{-1}$, which may correspond to a second planet.

properties of their planets will come into sharper focus. We are monitoring a sample of 147 late K and early M stars as part of the California Planet Survey at Keck Observatory with a current temporal baseline of $\approx 12 \mathrm{yr}$ and Doppler precision of $2-3 \mathrm{~m} \mathrm{~s}^{-1}$ (Johnson et al. 2007; Howard et al. 2010). In this contribution we announce the detection of a new Saturn-mass planet orbiting a nearby M dwarf. Gl 649 is only the eleventh M-type star known to harbor at least one Doppler-detected planet, ${ }^{10}$ and it is only the seventh low-mass star with a Doppler-detected giant planet (see also Bonfils et al. 2005; Maness et al. 2007; Forveille et al. 2009; Mayor et al. 2009, for examples of low-mass planet detections). In the following section we describe the stellar properties of $\mathrm{Gl}$ 649, and our spectroscopic observations and Doppler-shift measurements. In $\S 3$ we test the validity of our interpretation of the observed radial velocity (RV) variations by measuring the falsealarm probability and by examining our photometric measurements. We conclude in $\S 4$ with a summary and discussion of Gl 649b, and we place this latest exoplanet in context with other giant planets discovered around $\mathrm{M}$ dwarfs.

\section{OBSERVATIONS AND ANALYSIS}

\subsection{Stellar Properties}

Gl 649 (=HIP 83043) is an M1.5 dwarf with a Hipparcos parallax-based distance of $10.34 \pm 0.15 \mathrm{pc}$ (van Leeuwen 2007), apparent magnitude $V=9.7$, and absolute magnitude $M_{V}=9.627 \pm 0.053 .{ }^{11}$ We use the broadband metallicity calibration of Johnson \& Apps (2009) to estimate $[\mathrm{Fe} / \mathrm{H}]=$ $+0.08 \pm 0.06$, and we adopt the stellar mass estimate provided by the Delfosse et al. (2000) $K_{s}$-band mass-luminosity relationship, which gives $M_{\star}=0.54 \pm 0.05 M_{\odot}$. Using the infrared flux method, Alonso et al. (1996) give an effective temperature $T_{\text {eff }}=3700 \pm 60 \mathrm{~K}$. Wright et al. (2004) measured the emis-

\footnotetext{
${ }^{10}$ Several additional low-mass host stars have been discovered by gravitational microlensing surveys (Bond et al. 2004; Gould et al. 2006; Beaulieu et al. 2006; Dong et al. 2009, e.g.).

${ }^{11}$ Vizier Online Data Catalog, 1239 (ESA, 1997).
}

sion in the Ca II H emission line relative to the stellar photosphere on the Mount Wilson scale (Duncan et al. 1991) and give a median "grand $S$ " value of 1.55. This $S$ value places the chromospheric activity of Gl 649 among the top $20 \%$ of nearby early M-type stars, as shown by Rauscher \& Marcy (2006) and by Gizis et al. (2002). Our spectra show $\mathrm{H} \alpha$ to be in absorption, as was found for all Balmer lines observed in the spectrum of Gl 649 (Gizis et al. 2002). The stellar properties of Gl 649 are summarized in Table 2.

\subsection{Radial Velocities and Keplerian Fit}

We began monitoring Gl 649 at Keck Observatory in 1999 October using the High-Resolution Echelle spectrometer (Vogt et al. 1994) in our standard iodine cell setup with the B5 decker, giving a reciprocal resolution $\lambda / \Delta \lambda=55,000$ per $\sim 4$ pixel

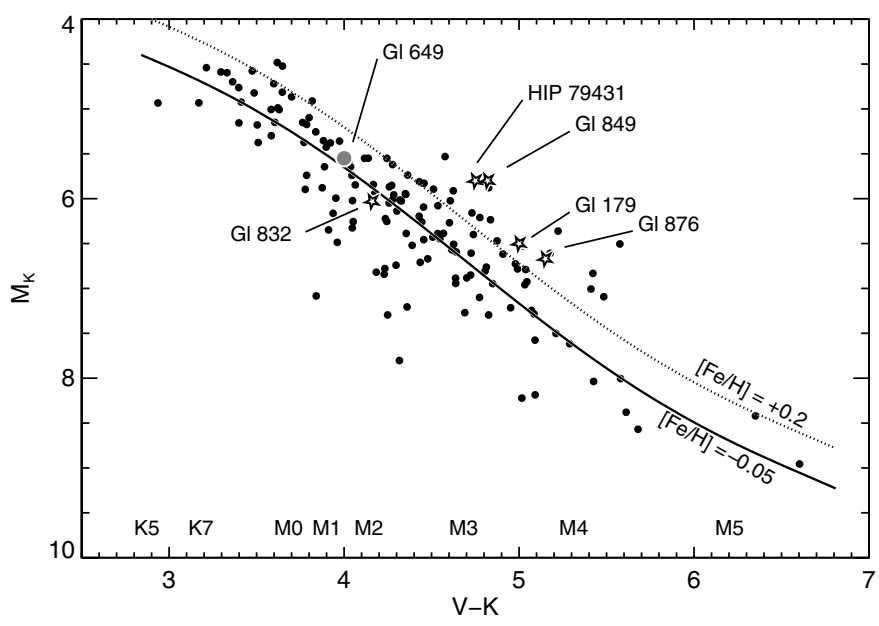

FIG. 1.-Our Keck sample of low-mass stars plotted in the $\left\{V-K, M_{K}\right\}$ plane. The solid line is a fifth-order polynomial fit to the mean main sequence for stars within $10 \mathrm{pc}$, which Johnson \& Apps (2009) identify as an isometallicity contour with $[\mathrm{Fe} / \mathrm{H}]$ equal to the mean value of the Solar neighborhood. The dotted line corresponds to $[\mathrm{Fe} / \mathrm{H}]=+0.2$ based on the calibration of Johnson \& Apps. The open stars show the positions of all of the M dwarfs known to harbor at least one giant planet. The filled circle denotes the position of Gl 649 . 
TABLE 2

Stellar Properties and Orbital SOlution fOR Gl 649

\begin{tabular}{|c|c|}
\hline Parameter & Value \\
\hline$V$ & $9.70 \pm 0.04$ \\
\hline$K \quad \ldots \ldots$. & $5.62 \pm 0.02$ \\
\hline$V-K \quad \ldots$ & $4.08 \pm 0.05$ \\
\hline$B-V \ldots \ldots$ & $1.52 \pm 0.04$ \\
\hline$M_{V} \quad \ldots \ldots$ & $9.63 \pm 0.05$ \\
\hline$M_{K} \quad \ldots \ldots$ & $5.55 \pm 0.02$ \\
\hline$d(\mathrm{pc}) \quad \ldots .$. & $10.34 \pm 0.15$ \\
\hline$M_{\star}\left(\mathrm{M}_{\odot}\right)$ & $0.54 \pm 0.05$ \\
\hline$T_{\text {eff }}(\mathrm{K}) \ldots$ & $3700 \pm 60$ \\
\hline$[\mathrm{Fe} / \mathrm{H}] \quad \ldots \ldots$ & $+0.08 \pm 0.06$ \\
\hline$P$ (days) $\ldots$. & $598.3 \pm 4.2$ \\
\hline$P(\mathrm{yr}) \ldots \ldots$. & $1.638 \pm 0.011$ \\
\hline$K\left(\mathrm{~m} \mathrm{~s}^{-1}\right) \ldots$ & $12.4 \pm 1.1$ \\
\hline$e \quad \ldots \ldots \ldots \ldots \ldots \ldots \ldots \ldots \ldots \ldots \ldots$ & $0.30 \pm 0.08$ \\
\hline$T_{P}($ Julian Date -2400000$) \quad \ldots \ldots \ldots$ & $12876 \pm 22$ \\
\hline$\omega(\operatorname{deg}) \quad \ldots \ldots \ldots \ldots \ldots \ldots \ldots$ & $352 \pm 15$ \\
\hline$M_{P} \sin i\left(M_{\text {Jup }}\right) \quad \ldots$ & $0.328 \pm 0.032$ \\
\hline 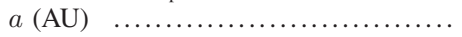 & $1.135 \pm 0.035$ \\
\hline$N_{\text {obs }} \ldots \ldots \ldots \ldots \ldots$ & 44 \\
\hline $\operatorname{rms}\left(\mathrm{ms}^{-1}\right) \quad \ldots \ldots \ldots \ldots \ldots \ldots \ldots \ldots \ldots \ldots \ldots$ & 4.2 \\
\hline 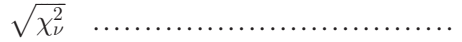 & 1.17 \\
\hline 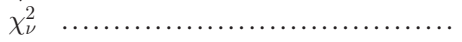 & 1.37 \\
\hline
\end{tabular}

resolution element (Howard et al. 2009). We measured the Doppler shifts of the star from each star-times-iodine observation using the standard analysis procedure presented by Butler et al. (1996), with subsequent improvements over the years. For HIRES observations made prior to the 2004 CCD upgrade, the measurement uncertainties range from $3.3-4.4 \mathrm{~m} \mathrm{~s}^{-1}$, and improve to $0.9-1.5 \mathrm{~m} \mathrm{~s}^{-1}$ thereafter.

Our 44 radial velocities are presented in Table 3 (without jitter) and the time series is shown in Figure 2 (with jitter). The scatter in the measurements is larger than expected from the measurement errors, and a periodogram analysis of the data reveals strong power at periods near 592 days, with a corresponding analytic false-alarm probability $<0.0001$ (Fig. 3).

We used the partially-linearized Keplerian fitting code RVLIN $^{13}$ described by Wright \& Howard (2009) to search for a best-fitting orbital solution to the data. To ensure proper weighting of our measurements in the fitting procedure, we inflated the error bars to account for RV noise from astrophysical sources. This stellar "jitter" term is calculated based on the star's chromospheric activity, $B-V$ color, and absolute $V$-band magnitude using the formula of Wright (2005). We adopt a jitter estimate of $3 \mathrm{~m} \mathrm{~s}^{-1}$ for Gl 649 , which we add in quadrature to the measurement errors.

\footnotetext{
${ }^{13}$ We use $\sqrt{\chi_{\nu}^{2}}$ to indicate the factor by which the observed scatter about the best-fitting model differs from our expectation based on the measurement errors. Thus, the scatter about our model is a factor of 1.17 larger than our average error bar.
}

TABLE 3

Radial Velocities For Gl 649

\begin{tabular}{|c|c|c|}
\hline $\mathrm{JD}-2,440,000$ & $\begin{array}{c}\mathrm{RV} \\
\left(\mathrm{m} \mathrm{s}^{-1}\right)\end{array}$ & $\begin{array}{c}\text { Uncertainty } \\
\left(\mathrm{m} \mathrm{s}^{-1}\right)\end{array}$ \\
\hline $11,409.824 \quad \ldots$ & -1.54 & 3.35 \\
\hline $11,705.913 \quad \ldots \ldots \ldots \ldots$ & 25.38 & 3.80 \\
\hline $12,004.048 \quad \ldots \ldots \ldots \ldots$ & -8.26 & 3.73 \\
\hline $12,007.010 \quad \ldots \ldots \ldots \ldots$ & -10.05 & 4.01 \\
\hline $12,008.001 \quad \ldots \ldots \ldots \ldots$ & -11.88 & 3.92 \\
\hline $12,009.070 \quad \ldots \ldots \ldots \ldots$ & -2.72 & 3.65 \\
\hline $12,097.968 \quad \ldots \ldots \ldots \ldots$ & -8.16 & 3.60 \\
\hline $12,098.912 \quad \ldots \ldots \ldots \ldots$ & 1.04 & 3.83 \\
\hline $12,099.844 \quad \ldots \ldots \ldots \ldots \ldots$ & 4.90 & 3.63 \\
\hline $12,100.895 \quad \ldots \ldots \ldots \ldots$ & 4.57 & 3.39 \\
\hline $12,127.911 \quad \ldots \ldots \ldots \ldots$ & 0.00 & 3.72 \\
\hline $12,161.808 \quad \ldots \ldots \ldots \ldots$ & 5.87 & 3.89 \\
\hline $12,189.748 \quad \ldots \ldots \ldots \ldots$ & 2.16 & 4.36 \\
\hline $12,390.092 \quad \ldots \ldots \ldots \ldots$ & 13.12 & 3.74 \\
\hline $12,486.759 \quad \ldots \ldots \ldots \ldots$ & -1.86 & 3.46 \\
\hline $12,514.756 \quad \ldots \ldots \ldots \ldots$ & -5.61 & 3.56 \\
\hline $12,535.722 \quad \ldots \ldots \ldots \ldots$ & -6.90 & 3.57 \\
\hline $12,574.690 \quad \ldots \ldots \ldots \ldots$ & 2.29 & 4.02 \\
\hline $12,712.157 \quad \ldots \ldots \ldots \ldots$ & -3.63 & 3.71 \\
\hline $12,777.039 \quad \ldots \ldots \ldots \ldots$ & 7.53 & 3.93 \\
\hline $12,804.920 \quad \ldots \ldots \ldots \ldots$ & 13.40 & 3.75 \\
\hline $12,832.961 \quad \ldots \ldots \ldots \ldots$ & 13.60 & 3.91 \\
\hline $13,179.964 \quad \ldots \ldots \ldots \ldots$ & -9.51 & 3.82 \\
\hline $13,195.812 \quad \ldots \ldots \ldots \ldots$ & 0.17 & 3.38 \\
\hline $13,430.124 \quad \ldots \ldots \ldots \ldots$ & 13.32 & 1.20 \\
\hline $13,547.904 \quad \ldots \ldots \ldots \ldots$ & 12.84 & 0.99 \\
\hline $13,842.077 \quad \ldots \ldots \ldots \ldots$ & -3.95 & 0.97 \\
\hline $13,934.875 \quad \ldots \ldots$ & 9.44 & 0.95 \\
\hline $14,640.044 \quad \ldots \ldots$ & 14.79 & 1.16 \\
\hline $14,671.928 \quad \ldots \ldots \ldots \ldots$ & 16.81 & 0.72 \\
\hline $14,673.897 \quad \ldots \ldots \ldots \ldots$ & 25.43 & 0.58 \\
\hline $14,779.698 \quad \ldots \ldots \ldots \ldots$ & 8.75 & 1.14 \\
\hline $14,964.087 \quad \ldots \ldots \ldots \ldots$ & 4.24 & 0.96 \\
\hline $14,985.917 \quad \ldots \ldots \ldots \ldots$ & -3.91 & 0.96 \\
\hline $15,014.851 \quad \ldots \ldots \ldots \ldots$ & -2.75 & 0.98 \\
\hline $15,015.879 \quad \ldots \ldots \ldots \ldots$ & -7.25 & 0.97 \\
\hline $15,019.036 \quad \ldots \ldots \ldots \ldots$ & -2.05 & 1.51 \\
\hline $15,041.950 \quad \ldots \ldots \ldots \ldots$ & -9.03 & 1.05 \\
\hline $15,042.786 \quad \ldots \ldots \ldots \ldots$ & -6.49 & 0.97 \\
\hline $15,043.900 \quad \ldots \ldots \ldots \ldots$ & -9.16 & 1.00 \\
\hline $15,048.857 \quad \ldots \ldots \ldots \ldots$ & -3.67 & 1.28 \\
\hline $15,075.744 \quad \ldots \ldots \ldots \ldots$ & 4.40 & 0.91 \\
\hline $15,111.736 \quad \ldots \ldots \ldots \ldots$ & -2.10 & 1.07 \\
\hline
\end{tabular}

We find that a single-planet Keplerian model with a period $P=598.3 \pm 4.2$ days, eccentricity $e=0.30 \pm 0.08$, and velocity semiamplitude $K=12.4 \pm 1.1 \mathrm{~m} \mathrm{~s}^{-1}$ results in a root mean square (rms) scatter of $4.2 \mathrm{~m} \mathrm{~s}^{-1}$ in the residuals and $\sqrt{\chi_{\nu}^{2}}=1.17$, indicating an acceptable fit. ${ }^{12}$ The resulting minimum planet mass is $M_{P} \sin i=0.328 M_{\mathrm{Jup}}$, and the semimajor axis is $a=1.135 \mathrm{AU}$. The best-fitting solution is shown in

\footnotetext{
${ }^{12}$ At http://exoplanets.org/code/.
} 


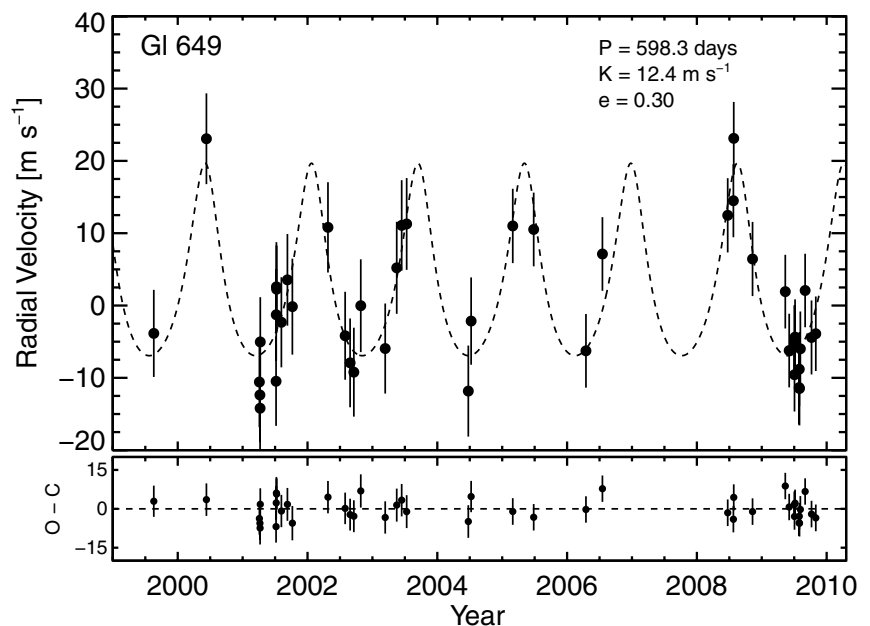

FIG. 2.-Our Keck/HIRES radial velocity time series for Gl 649. The dashed line shows the best-fitting Keplerian model. The rms scatter of the residuals (bottom panel) is $4.2 \mathrm{~m} \mathrm{~s}^{-1}$, and $\sqrt{\chi_{\nu}^{2}}=1.17$.

Figure 2, with the residuals to the fit shown in the lower panel. The orbital parameters are listed in Table 2.

The parameter uncertainties given here were estimated using a Markov Chain Monte Carlo (MCMC) algorithm with $10^{7}$ links, in which a single randomly-chosen parameter was perturbed at each link, with a perturbation size tuned such that $20 \%-40 \%$ of the jumps were executed (see, e.g., Ford 2005; Winn et al. 2008, and references therein). The resulting "chains" of parameters form the posterior probability distribution, from which we select the 15.9 and 84.2 percentile levels in the cumulative distributions (CDF) as the "one-sigma" confidence limits. In most cases, the posterior probability distributions were approximately Gaussian.

\section{TESTING THE NULL HYPOTHESIS}

\subsection{False-Alarm Probability}

The Doppler semiamplitude of our best-fitting model, $K=12.4 \mathrm{~m} \mathrm{~s}^{-1}$, is comparable to the measurement uncertainties and stellar jitter, which prompted us to test the nullhypothesis that the apparent periodicity arose by chance from larger-than-expected radial velocity fluctuations and sparse sampling. We tested this possibility calculating the false-alarm probability (FAP) based on the goodness of fit statistic $\Delta \chi_{\nu}^{2}$ (Howard et al. 2009; Marcy et al. 2005; Cumming 2004), which is the difference between two values of $\chi_{\nu}^{2}$ : one from the singleplanet Keplerian fit and one from the fit of a linear trend to the data. Each trial is constructed by keeping the times of observation fixed and scrambling the measurements, with replacement. We record the $\Delta \chi_{\nu}^{2}$ value after each trial and repeat this process for 10,000 trial data sets. For the ensemble set we compare the resulting distribution of $\Delta \chi_{\nu}^{2}$ to the value from the fit to the original data.

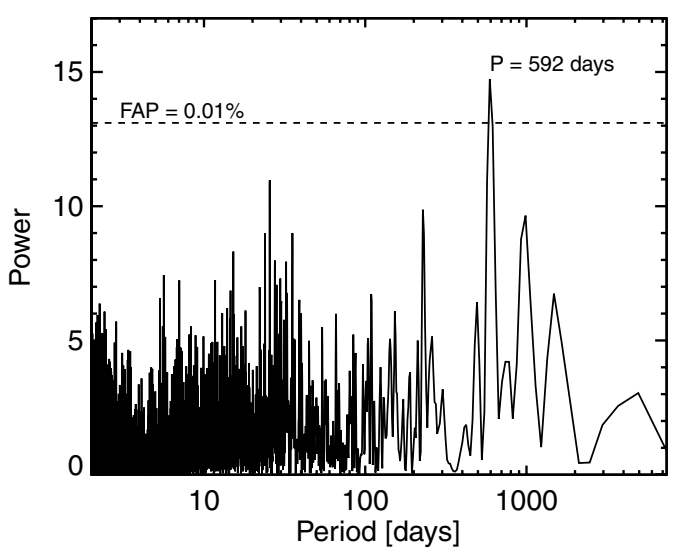

FIG. 3.-Periodogram analysis of our RV time series, which reveals a strong peak at $P=592$ days. The dashed lines show the analytic false-alarm probability of a peak arising from noise sampled at our times of observation.

We found that none of the $10^{4}$ trials resulted in a higher value of $\Delta \chi_{\nu}^{2}$, which we interpret as a $<0.0001$ probability that the 600 day periodicity is a spurious signal due to random fluctuations.

\subsection{Photometric Variability}

We note that our FAP value only addresses the existence of a periodicity in the radial velocities, but does not test its cause. As an additional test of the null hypothesis, we acquired brightness measurements of Gl 649 in the Johnson $V$ passband with the T3 $0.4 \mathrm{~m}$ automatic photometric telescope (APT) at Fairborn Observatory. The APT observations cover five observing seasons between 2001 February and 2009 June and reveal photometric variability in Gl 649 on both rotational and decadal timescales. Details on APT operations, data acquisition and reduction procedures, and precision of the observations can be found in Henry et al. (1995a, 1995b); Fekel et al. (2005); Eaton et al. (2003).

Our 337 Gl 649-minus-comparison $(V-C)$ differential magnitudes are plotted against heliocentric Julian Date in the top panel of Figure 4. The comparison star is HD 152342 $(V=7.10, B-V=0.35, \mathrm{~F} 2 \mathrm{~V})$. Most obvious in this plot are the year-to-year changes in the mean magnitude of the observations. The mean magnitudes have a range of 0.0126 mag and suggest the possible existence of a spot (magnetic) cycle in G1 649 with a length of at least several years (see, e.g., Henry 1999; Hall et al. 2009). The top panel also shows that the range in the $V-C$ observations is $\sim 0.02 \mathrm{mag}$ within all five observing seasons. The standard deviations of the individual five seasons are all between $0.0057 \mathrm{mag}$ and $0.0063 \mathrm{mag}$.

The check-minus-comparison $(K-C)$ differential magnitudes are plotted in the second panel of Figure 4 at the same magnitude scale as the top panel. The check star is HD 153897 $(V=6.57, B-V=0.43, \mathrm{~F} 4 \mathrm{~V})$. The yearly means of the 

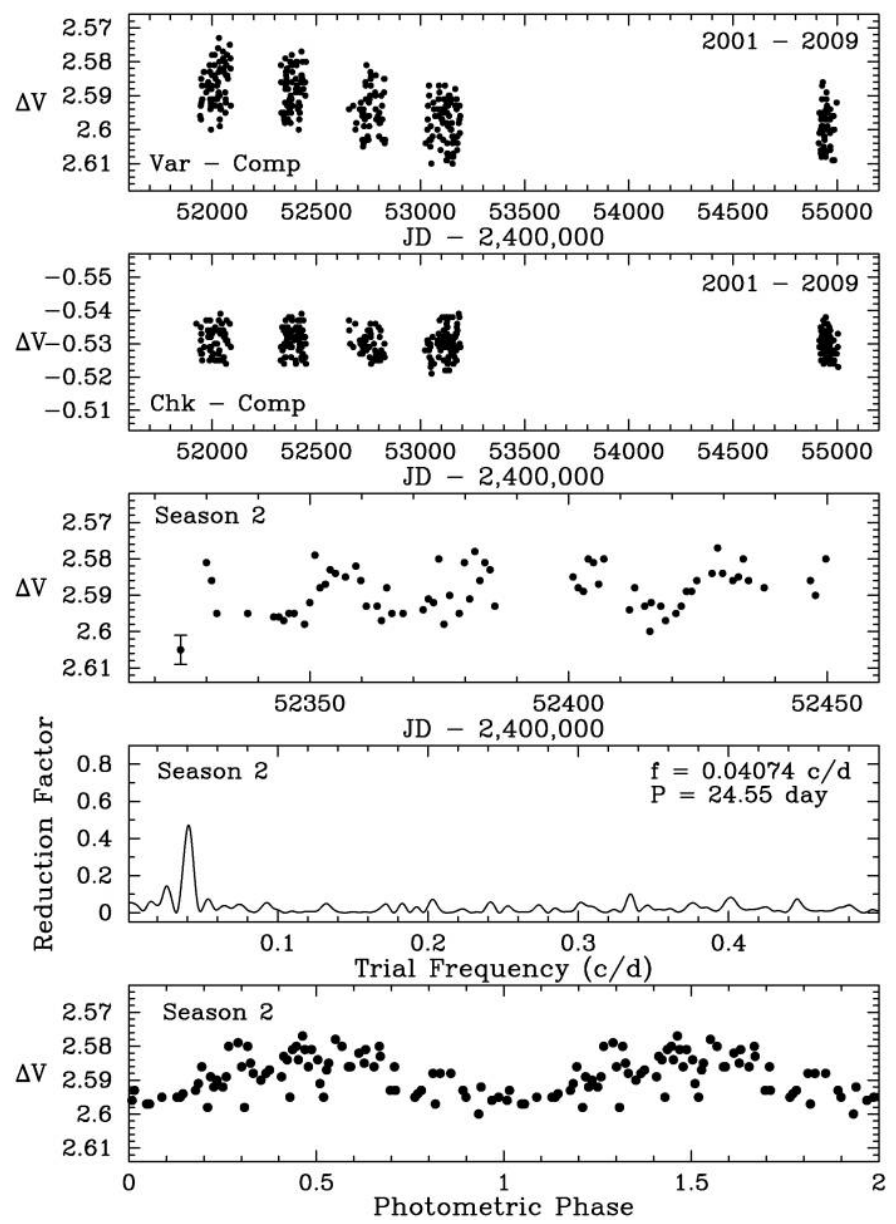

FIG. 4.-Top panel: Our $337 V-C$ photometric observations of Gl 649 in the Johnson $V$ band acquired with the T3 $0.4 \mathrm{~m}$ APT at Fairborn Observatory. Second panel: The $K-C$ observations plotted with an identical scale as the top panel. Comparison of these two data sets shows that Gl 649 varies in brightness on night-to-night and year-to-year timescales. Third panel: Observations from the second observing season plotted on an expanded $x$-axis clearly show lowamplitude brightness variability in Gl 649. Fourth panel: Frequency spectrum of the observations from season 2 gives a best period of 24.5 days. Bottom panel: Plot of the data from season 2 phased with the 24.55 day period reveals coherent variability with a peak-to-peak brightness amplitude $0.012 \mathrm{mag}$.

$K-C$ measurements have a range of only $0.0016 \mathrm{mag}$; their standard deviation is only $0.0007 \mathrm{mag}$. The standard deviations of the individual five seasons are all between 0.0034 mag and 0.0042 mag. Thus, the larger night-to-night scatter of the $V-C$ measurements and the observed year-to-year change in the $V-C$ mean magnitudes must both be intrinsic to Gl 649 .

The observations from season 2 are replotted in the third panel of Figure 4, again on the same magnitude scale as in panels 1 and 2. Low-amplitude variability is clearly seen with a period of 20-30 days. Panel 4 shows our computed frequency spectrum for season 2, where the y axis plots the reduction factor in the variance of the observations for each trial frequency (Vaníček 1971). We find a clear period of 24.55 days; the observations are plotted phased with this period in the bottom panel of Figure 4. A least-squares sine fit to the phase curve gives a peak-to-peak amplitude of $0.0117 \pm 0.0015 \mathrm{mag}$. Similar analyses yield periods of 23.72, 27.92, 25.80, and 21.86 days for seasons 1, 3, 4, and 5, respectively. The mean of these five periods is $24.8 \pm 1.0$ days, which we take to be the rotation period of Gl 649 revealed by rotational modulation in the visibility of cool starspots on the photosphere of Gl 649. This photometric variability is consistent with the level of chromospheric activity in the star, as mentioned above.

Henry et al. (1995b) show many examples of active stars with low-amplitude starspot variability. Queloz et al. (2001) and Paulson et al. (2004) show several examples of stellar spots masquerading as planets. In the case of Gl 649 described here, the photometric observations reveal variability timescales that are inconsistent with the 600 day radial velocity variations. Sinilarly, we examined the time-variability chromospheric emission from each of our spectroscopic observations. While the $S$ value, as measured from the $\mathrm{Ca} \mathrm{II} \mathrm{H}$ and $\mathrm{K}$ emission, has a variance of 0.14 dex, we observed no periodicities near the putative orbital period. The lack of photometric and chromospheric variability provide additional strong support for the interpretation of planetary reflex motion as the cause of the observed radial velocity variability in Gl 649 .

\section{DISCUSSION}

We have presented the discovery of a Saturn-mass planet $\left(M_{P} \sin i=0.328 M_{\text {Jup }}\right)$ orbiting the nearby, low-mass star Gl $649\left(d=10.34 \mathrm{pc}, 0.54 M_{\odot}\right)$. Gl 649b resides in an eccentric $(e=0.30)$ orbit with a period of 598.3 days, corresponding to a semimajor axis $a=1.135 \mathrm{AU}$.

Gl 649 is only the seventh M dwarf known with a Dopplerdetected giant planet, and the fifth detection from among the 147 low-mass stars we have monitored over the past decade at Keck Observatory (e.g., Johnson et al. 2007). The low Doppler amplitude of the planet $\left(K=12.4 \mathrm{~m} \mathrm{~s}^{-1}\right)$ highlights our need to attain high measurement precision to find low-mass planets, and to maintain that precision over long time baselines to detect planets at larger semimajor axes.

Johnson et al. (2007) recently analyzed the detection rate among our sample of low-mass stars and reported a $1.8 \%$ occurrence rate of planets with $a<2.5 \mathrm{AU}$. The increased time baseline and new detections of our sample suggest that a reanalysis of the frequency of planets around $\mathrm{M}$ dwarfs is warranted. Following Johnson et al. (2007), we first note that the $\approx 10 \mathrm{yr}$ time baseline of our survey, together with our radial velocity precision, provides us with sensitivity to planets with $M_{P} \sin i \gtrsim 0.3 M_{\text {Jup }}$ out to semimajor axes $a \approx 2.5 \mathrm{AU}$, assuming an average stellar mass $M_{\star}=0.5 M_{\odot}$. Note that in the analysis that follows, we exclude the recently detected planets Gl 832b, which was discovered by the Anglo-Australian Observatory planet search (Bailey et al. 2008), and HIP 79431b, which was only recently added to the Keck survey as part of the 
metallicity-biased M-to-K program (Apps et al. 2010, submitted).

The probability density function (PDF) for the fraction of stars with planets, given our number of detections $k=5$ and total sample size $N=147$, is given by the binomial distribution $P(f \mid k, N) \propto f^{5}(1-f)^{147-5}$. The overall occurrence rate from our sample is given by the maximum of the PDF, which we measure to be $f=3.4_{-0.9}^{+2.2} \%$, where the upper and lower limits represent the $68.3 \%$ confidence interval measured from the cumulative distribution function.

The corresponding giant planet fraction around Sunlike stars was recently measured by Cumming et al. (2008, cf. their Table 1), who report $f=7.6 \pm 1.3 \%$. In a similar study, Bowler et al. (2010) measured the planet fraction around stars with $M_{\star}>1.5 M_{\odot}$ to be $26_{-8}^{+9} \%$, albeit for minimum masses $M_{P} \sin i \gtrsim 1 M_{\mathrm{Jup}}$. Thus, the detection rate of giant planets around $\mathrm{M}$ dwarfs consistently lags behind that of higher mass stars, despite the enhanced detectability of planets around less massive stars since $K \propto M_{\star}^{-2 / 3}$ for a fixed planet mass and period. The contrast between the measured planet fractions between $\mathrm{M}$ dwarfs and massive stars points to an even stronger correlation between stellar mass and planet occurrence than measured by Johnson et al. (2007).

The correlation between stellar mass and planet formation is an important piece of observational evidence in support of the core accretion model of planet formation. In this model, giant planets form in a bottom-up process, starting with the collisions of small dust grains and proceeding up through the formation of large protoplanetary cores (see Ida \& Lin 2004; Alibert et al. 2005 , for reviews). Once these cores attain a critical mass of $\sim 10 M_{\oplus}$, they can rapidly accrete gas from the surrounding disk. Given the limited lifetime of the gas disk, which dissipates on timescales shorter than 5 Myr (Hernández et al. 2008; Currie et al. 2009), the formation of gas giant planets is a race against time that is rarely won in the protoplanetary disks of low-mass stars. The low density of raw materials, low orbital frequencies $\left(\Omega \propto 1 / P \propto M_{\star}^{1 / 2}\right.$ at fixed $\left.a\right)$, and unfavorable temperature profiles in the disks around M-type stars greatly inhibit the core growth, which results in a lower occurrence of giant planets (Laughlin et al. 2004; Ida \& Lin 2005; Kennedy \& Kenyon 2008; Dodson-Robinson et al. 2009).

Another important predictor of planet occurrence is stellar metallicity. Fischer \& Valenti (2005b) showed that the fraction of Sunlike stars with planets correlates strongly with $[\mathrm{Fe} / \mathrm{H}]$, with an occurrence rate of $\sim 3 \%$ for $[\mathrm{Fe} / \mathrm{H}]<0$ and a rise to $\approx 25 \%$ for $[\mathrm{Fe} / \mathrm{H}]>+0.3$. Until recently, it was difficult to properly account for metallicity among the $\mathrm{M}$ dwarfs because the LTE spectral analysis tools used for more massive stars are not amenable to the complex spectra of low-mass stars (Maness et al. 2007). Because of the lack of knowledge about the metallicity distribution of $\mathrm{M}$ dwarfs in general, and low-mass stars with planets in particular, it was difficult to determine whether stellar mass or metallicity lay at the root cause of the puacity of planets around $\mathrm{M}$ dwarfs.

The mass/metallicity issue was recently addressed by Johnson \& Apps (2009), who derived a revised broadband photometric metallicity calibration for $\mathrm{M}$ dwarfs. They examined a sample of $\mathrm{M}$ dwarfs with F, G, and $\mathrm{K}$ wide binary companions. By anchoring the metallicity of the M dwarf to its earlier-type companion, Johnson \& Apps observed that metal-rich M stars reside "above" the mean main sequence of the solar neighborhood when viewed in the $\left\{V-K, M_{K}\right\}$ plane. Further, they noticed the majority of the 7 planetary systems (containing planets of all masses) that were known at the time contain metal-rich host stars.

Figure 1 shows that with the addition of 3 new planethost stars since the study of Johnson \& Apps (2009), the planetmetallicity correlation among $\mathrm{M}$ dwarfs appears to persist. We can quantify this relationship by examining the fraction of stars in our Keck survey with $[\mathrm{Fe} / \mathrm{H}] \geq 0$ that harbor giant planets. Including Gl 649, we find that all four of the stars that harbor at least one giant planet ${ }^{14}$ fall within the subsamlple of 80 targets with $[\mathrm{Fe} / \mathrm{H}] \geq 0$. Based on this, we measure a planet fraction $f=5.5_{-2.1}^{+2.7} \%$ for $[\mathrm{Fe} / \mathrm{H}]>0$. If we restrict our analysis to $[\mathrm{Fe} / \mathrm{H}]>+0.2$ (dashed line in Fig. 1), 3 of these 33 "super metal-rich" stars harbor planets, corresponding to $f=10.7_{-4.2}^{+5.9 \%}$.

The uncertainties in our measured planet fractions are large due to the small sample sizes involved. This underscores the need for extending the time baseline of our current survey and expanding the target list to include additional low-mass stars. Future surveys of nearby, low-mass stars such as the M2K planet search (Apps et al. 2010) and the MEarth transit survey (Irwin et al. 2009) will build upon the current sample and provide a clearer picture of the planet-metallicity relationship suggested from our analysis. A larger sample of planets detected around $\mathrm{M}$ dwarfs will also provide crucial leverage in understanding the relationship between stellar mass and planet properties, especially when compared to the growing sample of planets discovered around massive stars with $M_{\star}>1.5 M_{\odot}$.

We thank the many observers who contributed to the velocities reported here. We gratefully acknowledge the efforts and dedication of the Keck Observatory staff, especially Grant Hill and Scott Dahm for support of HIRES and Greg Wirth for support of remote observing. We are also grateful to the time assignment committees of NASA, NOAO, and the University of California for their generous allocations of observing time. We acknowledge R. Paul Butler and S. S. Vogt for many years of contributing to the data presented here. A. W. H. gratefully

\footnotetext{
${ }^{14}$ We exclude Gl317 in this analysis because it lacks a reliable parallax measurement and cannot be accurately placed in the $\left\{V-K, M_{K}\right\}$ plane. The existing parallaxes given in the literature suggest that Gl 317 resides above the main sequence $\Delta M_{K}=0.08-0.37$, indicating either a Solar composition or that the star is metal-rich $(0.0 \lesssim[\mathrm{Fe} / \mathrm{H}] \lesssim+0.15)$.
} 
acknowledges support from a Townes Postdoctoral Fellowship at the University of California, Berkeley Space Sciences Laboratory. J. A. J. thanks the NSF Astronomy and Astrophysics Postdoctoral Fellowship program for support in the years leading to the completion of this work, and acknowledges support from NSF grant AST-0702821. G. W. M. acknowledges NASA grant NNX06AH52G. J. T. W. received support from NSF grant
AST-0504874. G. W. H. acknowledges support from NASA, NSF, Tennessee State University, and the State of Tennessee through its Centers of Excellence program. Finally, we wish to extend special thanks to those of Hawai'ian ancestry on whose sacred mountain of Mauna Kea we are privileged to be guests. Without their generous hospitality, the Keck observations presented herein would not have been possible.

\section{REFERENCES}

Alibert, Y., et al. 2005, A\&A, 434, 343

Alonso, A., Arribas, S., \& Martinez-Roger, C. 1996, A\&AS, 117, 227

Apps, K., et al. 2010, preprint (arXiv1001.1174

Bailey, J., et al. 2008, preprint (arxiv:0809.0172) 2009, ApJ, 690, 743

Beaulieu, J.-P., et al. 2006, Nature, 439, 437

Bond, I. A., et al. 2004, ApJ, 606, L155

Bonfils, X., et al. 2005, A\&A, 443, L15

Bowler, B., et al. 2010, ApJ, 709, 396

Butler, R. P., et al. 2006, PASP, 118, 1685 1996, PASP, 108, 500

Cumming, A. 2004, MNRAS, 354, 1165

Cumming, A., et al. 2008, PASP, 120, 531

Currie, T., et al. 2009, ApJ, 698, 1

Delfosse, X., et al. 2000, A\&A, 364, 217

Dodson-Robinson, S. E., et al. 2009, ApJ, 707, 79

Dong, S., et al. 2009, ApJ, 695, 970

Duncan, D. K., et al. 1991, ApJS, 76, 383

Eaton, J. A., Henry, G. W., \& Fekel, F. C. 2003, in The New Millennium. Volume II-The Telescopes We Use (Dordrecht: Kluwer) 189

Endl, M., et al. 2003, AJ, 126, 3099

Fekel, F. C., Henry, G. W., \& Lewis, C. 2005, AJ, 130, 794

Fischer, D. A., \& Valenti, J. 2005a, ApJ, 622, 1102 2005b, ApJ, 622, 1102

Ford, E. B. 2005, AJ, 129, 1706

Forveille, T., et al. 2009, A\&A, 493, 645

Gizis, J. E., Reid, I. N., \& Hawley, S. L. 2002, AJ, 123, 3356

Gould, A., et al. 2006, ApJ, 644, L37

Hall, J. C., et al. 2009, AJ, 138, 312

Henry, G. W. 1999, PASP, 111, 845
Henry, G. W., et al. 1995a, ApJS, 97, 513

Henry, G. W., Fekel, F. C., \& Hall, D. S. 1995b, AJ, 110, 2926

Hernández, J., et al. 2008, ApJ, 686, 1195

Howard, A. W., et al. 2009, ApJ, 696, 75

Howard, A. W., et al. 2010, ApJ, submitted

Ida, S., \& Lin, D. N. C. 2004, ApJ, 604, 388 2005, ApJ, 626, 1045

Irwin, J., et al. 2009, ApJ, 701, 1436 2008, preprint arxiv:0807.1316

Johnson, J. A., \& Apps, K. 2009, ApJ, 699, 933

Johnson, J. A., et al. 2007, ApJ, 670, 833

Kennedy, G. M., \& Kenyon, S. J. 2008, ApJ, 673, 502

Laughlin, G., Bodenheimer, P., \& Adams, F. C. 2004, ApJ, 612, L73

Maness, H. L., et al. 2007, PASP, 119, 90

Marcy, G., et al. 2005, Progr. Theoretical Phys. Suppl., 158, 24

Mayor, M., et al. 2009, A\&A, 507, 487

Nielsen, E. L., \& Close, L. M. 2009, preprint (ArXiv:0909.4531)

Paulson, D. B., et al. 2004, AJ, 127, 1644

Pravdo, S. H., \& Shaklan, S. B. 2009, ApJ, 700, 623

Queloz, D., et al. 2001, A\&A, 379, 279

Rauscher, E., \& Marcy, G. W. 2006, PASP, 118, 617

Rivera, E. J., et al. 2005, ApJ, 634, 625

van Leeuwen, F. 2007, A\&A, 474, 653

Vaníček, P. 1971, Ap\&SS, 12, 10

Vogt, S. S., et al. 1994, Proc. SPIE Instrumentation in Astronomy VIII, ed. D. L. Crawford, \& E. R. Craine, 2198, 362

Winn, J. N., et al. 2008, ApJ, 683, 1076

Wright, J. T. 2005, PASP, 117, 657

Wright, J. T., \& Howard, A. W. 2009, ApJS, 182, 205

Wright, J. T., et al. 2004, ApJS, 152, 261 